\title{
Evidence for a critical contribution of haploinsufficiency in the complex pathogenesis of Marfan syndrome
}

\author{
Daniel P. Judge, ${ }^{1}$ Nancy J. Biery, ${ }^{2}$ Douglas R. Keene, ${ }^{3}$ Jessica Geubtner, ${ }^{1}$ Loretha Myers, ${ }^{2}$ \\ David L. Huso, ${ }^{4}$ Lynn Y. Sakai, ${ }^{3}$ and Harry C. Dietz ${ }^{2,5}$

\begin{abstract}
1Division of Cardiology and 2McKusick-Nathans Institute of Genetic Medicine, Johns Hopkins University, Baltimore, Maryland, USA.
\end{abstract} \\ ${ }^{3}$ Shriners Hospital for Children, Oregon Health and Science University, Portland, Oregon, USA. ${ }^{4}$ Department of Comparative Medicine, \\ Johns Hopkins University, Baltimore, Maryland, USA. ${ }^{5}$ Howard Hughes Medical Institute, Bethesda, Maryland, USA.
}

\begin{abstract}
Marfan syndrome is a connective tissue disorder caused by mutations in the gene encoding fibrillin-1 (FBN1). A dominant-negative mechanism has been inferred based upon dominant inheritance, mulitimerization of monomers to form microfibrils, and the dramatic paucity of matrix-incorporated fibrillin-1 seen in heterozygous patient samples. Yeast artificial chromosome-based transgenesis was used to overexpress a disease-associated mutant form of human fibrillin-1 (C1663R) on a normal mouse background. Remarkably, these mice failed to show any abnormalities of cellular or clinical phenotype despite regulated overexpression of mutant protein in relevant tissues and developmental stages and direct evidence that mouse and human fibrillin-1 interact with high efficiency. Immunostaining with a human-specific $m A b$ provides what we believe to be the first demonstration that mutant fibrillin-1 can participate in productive microfibrillar assembly. Informatively, use of homologous recombination to generate mice heterozygous for a comparable missense mutation (C1039G) revealed impaired microfibrillar deposition, skeletal deformity, and progressive deterioration of aortic wall architecture, comparable to characteristics of the human condition. These data are consistent with a model that invokes haploinsufficiency for WT fibrillin-1, rather than production of mutant protein, as the primary determinant of failed microfibrillar assembly. In keeping with this model, introduction of a WT FBN1 transgene on a heterozygous C1039G background rescues aortic phenotype.
\end{abstract}

\section{Introduction}

Marfan syndrome (MFS) is an autosomal dominant systemic disorder of connective tissue affecting approximately 1 in 10,000 individuals $(1,2)$. Cardinal manifestations include aortic aneurysm and dissection, ocular lens dislocation, and long bone overgrowth. While current therapeutic interventions, such as the use of $\beta$-blockers to decrease hemodynamic stress and prophylactic surgical repair of the aortic root, have increased life expectancy, significant morbidity and early mortality remain associated with this condition (3). At best, $\beta$-blocker therapy slows the rate of aortic root growth but does not preclude the need for reconstructive surgery, which can require sequential revisions and often mandates life-long use of anticoagulants (3). Virtually no prophylactic therapies are available to modulate the progression of pulmonary, orthopedic, and ocular disease, or myxomatous degeneration and malfunction of the atrioventricular valves. Thus, there is ample opportunity to improve the quality of life in affected patients. In 1991, mutations in the gene (FBN1) encoding fibrillin-1 were found to cause MFS, and there is no demonstrated locus heterogeneity in classic forms (4-6). An improved understanding of fibrillin-1 metabolism and its role in tissue morphogenesis, growth, and homeostasis may offer the first real

Nonstandard abbreviations used: calcium-binding epidermal growth factor (cbEGF); embryonic day (ED); Marfan syndrome (MFS); mutant transgene 1-3 (Tg[mut1-3]); neomycin-resistance cassette (NeoR); vascular smooth muscle cell (VSMC); WT transgene (Tg[WT]); yeast artificial chromosome (YAC).

Conflict of interest: The authors have declared that no conflict of interest exists.

Citation for this article: J. Clin. Invest. 114:172-181 (2004)

doi:10.1172/JCI200420641. opportunity for primary prevention of selected manifestations of MFS and related disorders.

Several observations suggested a traditional dominant-negative mechanism in the pathogenesis of MFS. In this view, abnormal protein derived from the mutant allele interacts and interferes with protein derived from the normal allele, culminating in substantial loss of function. Supportive evidence includes (a) autosomal dominant inheritance, (b) aggregation of fibrillin-1 molecules to form complex extracellular structures termed microfibrils (7), and (c) the dramatic paucity of extracellular microfibrils in patient-derived tissues, far less than the $50 \%$ level predicted from simple loss of contribution from the mutant allele (8-10). This deficiency could be recapitulated by either immunohistochemical or pulse-chase analysis of cultured dermal fibroblasts, suggesting that dominant-negative interference is not restricted to enhanced proteolytic clearance of mutant microfibrils over time, but rather could occur at the level of microfibrillar assembly (9, 11-13).

While most patients with MFS harbor heterozygous missense mutations (14), another line of evidence in support of a traditional dominant-negative model stems from clinical characterization of patients harboring nonsense alleles. The predicted and observed consequence of premature termination codon mutations is the rapid and efficient clearance of the mutant transcripts via the nonsense-mediated mRNA decay pathway, precluding the production of substantial amounts of mutant fibrillin-1 $(15,16)$. Interestingly, in keeping with the dominant-negative hypothesis, many nonsense alleles associate with mild clinical presentations that can fail to meet diagnostic criteria for MFS $(17,18)$. Some patients with unstable mutant transcripts, however, have classic 
and severe disease (18-20). This raises two possibilities. Either haploinsufficiency per se can initiate the pathogenetic sequence of MFS or the truncated N-terminal peptides encoded by selected nonsense alleles have potent dominant-negative activity, allowing effective neutralization of function of the WT protein despite their low-level abundance. The lack of critical reagents needed to follow the fate of mutant fibrillin-1 and to comprehensively assess the in vivo consequence of heterozygous mutations has frustrated definitive resolution of this issue.

Prior murine models of MFS harbor targeted hypomorphic alleles of Fbn1 and showed classic phenotypic manifestations of MFS when bred to homozygosity $(21,22)$. The absence of overt manifestations in the heterozygous state has been attributed to the reduced (but not eliminated) expression from the targeted allele induced by transcriptional interference from the neomycin-resistance cassette (NeoR) (22). Although informative regarding the ability of murine models to clinically manifest fibrillin-1 deficiency, these mice do not provide the reliable means to test for dominant-negative interference imposed by the protein product of the mutant allele.

To test the hypothesis that dominant-negative interference drives phenotypic manifestations in MFS, yeast artificial chromosome-based (YAC-based) transgenesis was used to introduce expression of either WT or mutant human fibrillin-1 on a normal mouse background. Use of YACs allowed inclusion of all promoter elements, thus optimizing potential for physiologic temporal and spatial regulation of gene expression. The mutant transgene harbors a naturally occurring human mutation (C1663R) that substitutes one of six predictably spaced cysteine residues in a calcium-binding epidermal growth factor-like (cbEGF-like) domain, representative of the most common class of mutations causing MFS (14). These mice have no apparent clinical or histologic vascular phenotype despite high-level production of mutant protein (from 100 to $200 \%$ of the output of both endogenous mouse alleles among the different lines developed). Immunohistochemistry using a human fibrillin-1-specific mAb demonstrated abundant distribution of human epitope in microfibrillar beds deposited by cells transgenic for either the WT or mutant transgene, documenting both the bioavailability and competence of cysteine-substituted fibrillin-1 to participate in microfibrillar assembly. In contrast, the dramatic paucity of microfibrillar deposition and the presence of skeletal and cardiovascular abnormalities in a new engineered strain of mice harboring a cbEGF domain cysteine substitution $(\mathrm{C} 1039 \mathrm{G})$ in an endogenous allele reveals the ability of mouse models to clinically manifest the influence of heterozygous missense mutations, analogous to the situation in affected humans. Introduction of the WT transgene onto the $\mathrm{C} 1039 \mathrm{G}$ heterozygous background rescues the vascular phenotype in this mouse model of MFS. Taken together, these data are most consistent with a pathogenetic model that invokes reduced dosage of WT protein (rather than the presence of mutant protein) as the basis for impaired microfibrillar assembly. In contrast to a traditional dominant-negative effect, this view suggests that boosting fibrillin-1 expression holds promise for the treatment of patients with MFS.

A

B

C

\section{Results}

Expression of WT and mutant human fibrillin-1 in transgenic mice. To directly test the dominant-negative model of pathogenesis in MFS, we used YAC-based transgenesis to express either WT or mutant human fibrillin-1 on a normal mouse background (i.e., two functional Fbn1 alleles; Figure 1A) with proper regulation of gene expression. Human transgenes were used to provide an opportunity to specifically assess the fate of mutant protein, which is not possible in heterozygous human samples, using an $\mathrm{Ab}$ specific for human fibrillin-1. The mutant YAC transgene was engineered to produce fibrillin-1 with a cysteine substitution in a cbEGF-like domain (C1663R). This particular mutation had been described in a patient with a classic form of the disease $(23,24)$. Prior immunohistochemical and pulse-chase analyses of cultured dermal fibroblasts from this patient revealed impaired microfibrillar deposition despite normal fibrillin-1 synthesis, as is typically seen in MFS (24).

$\operatorname{Tg}(\mathrm{WT})$

FBN1 ${ }^{\text {max }}$ IIIIII

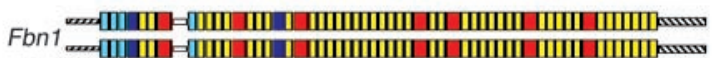
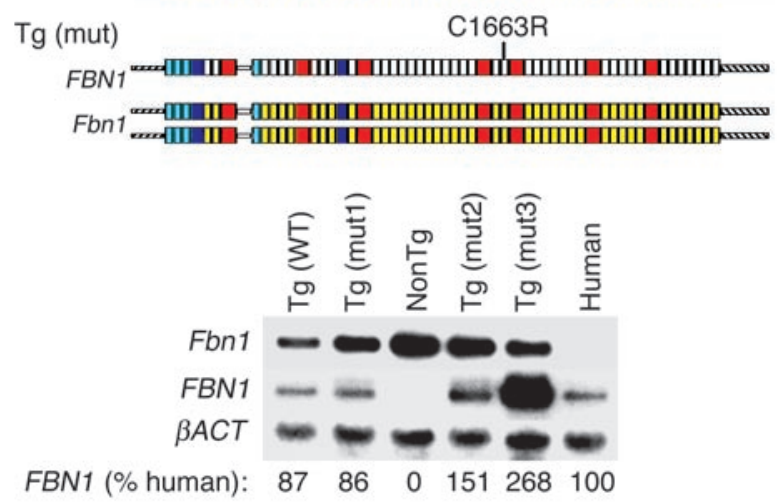

FBN1 (\% human):
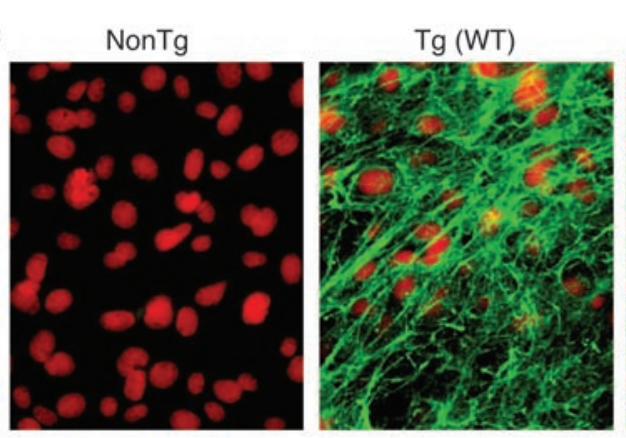

$\operatorname{Tg}$ (mut3)

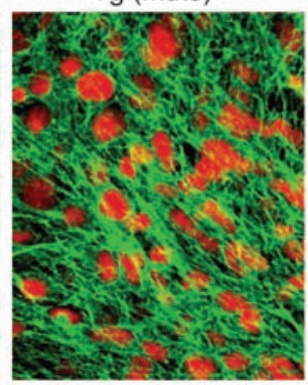

\section{Figure 1}

(A) Schematic representation of YAC transgenics. The endogenous untargeted mouse alleles (Fbn1) are shown with full-length WT human FBN1 or human FBN1 harboring a mutation (mut) that results in classic MFS in humans (C1663R). The cbEGF-like domains are shown in yellow (mouse) or white (human); 8-cys LTBP-binding domains are shown in red, and hybrid domains are shown in blue. (B) Northern blot analysis was performed on cultured dermal fibroblasts isolated from each of the transgenic strains. The radiolabeled probes recognize the $3^{\prime}$ UTRs of Fbn1 or FBN1. Expression of the genes encoding fibrillin-1 is normalized to expression of the gene encoding $\beta$-actin ( $\beta A C T)$ and compared with the amount of $F B N 1$ expression in a human fibroblast cell line isolated from a WT control individual. NonTg, nontransgenic. (C) Cultured dermal fibroblasts stained with $\mathrm{mAb}$ to human fibrillin-1. The left panel is from a littermate nontransgenic mouse; $\mathrm{Tg}(\mathrm{WT})$ is from a mouse with YAC containing WT FBN1; $\mathrm{Tg}$ (mut3) is from a mouse with YAC containing mutant FBN1 (C1663R). 
A
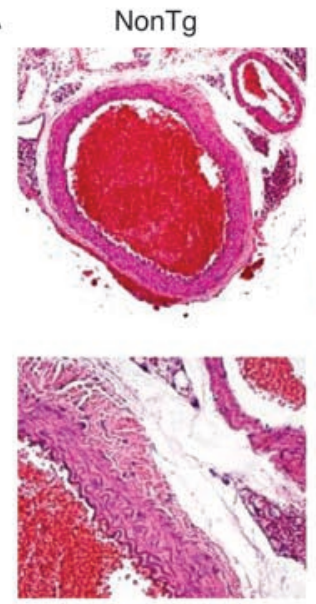

$\operatorname{Tg}(\mathrm{WT})$
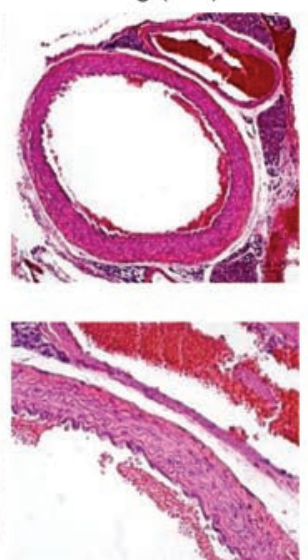

$\operatorname{Tg}($ mut3)
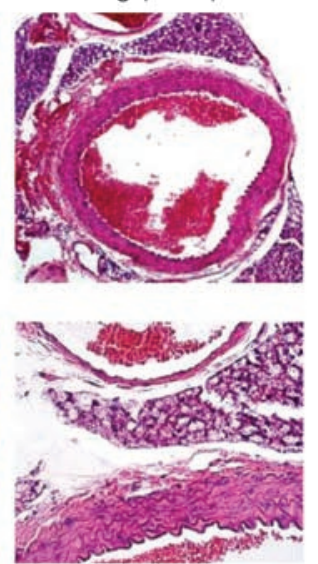

B

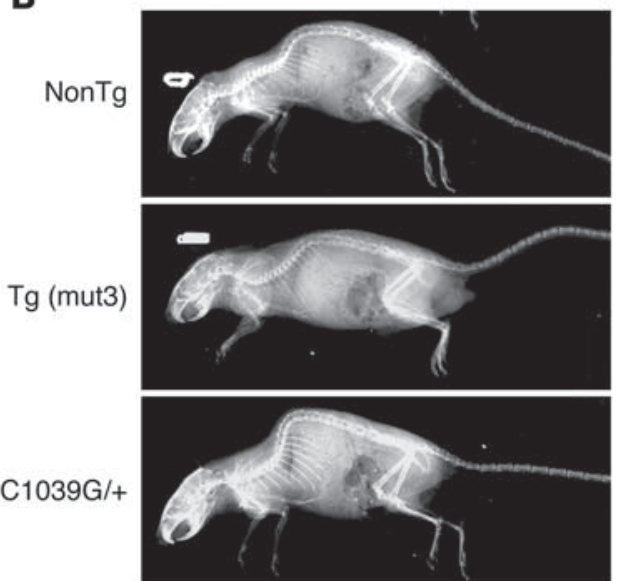

\section{Figure 2}

(A) Representative aortic wall sections from mice aged 12 months, stained with H\&E. NonTg is the nontransgenic control, Tg(WT) is from the strain expressing WT FBN1, and $\mathrm{Tg}$ (mut3) is from the strain with the highest expression of C1663R-mutant FBN1. Magnification, $\times 10$ (top) and $\times 40$ (bottom). In the low-power views, the adjacent pulmonary artery is visible. No differences were detected. (B) Representative radiographs taken of mice at 1 year of age. Overgrowth of the ribs and kyphoscoliosis are evident in the C1039G heterozygote (C1039G/+) mice (bottom), without discernible bony abnormalities in the $\mathrm{Tg}$ (mut3) mice, in comparison with non $\mathrm{Tg}$ control mice.

Three independent transgenic founder lines were established for the mutant transgene, termed $\operatorname{Tg}($ mut1-3), and one founder line was established for the WT transgene, termed $\mathrm{Tg}(\mathrm{WT})$. Quantitative Northern blot analysis of cultured dermal fibroblasts derived from these mice demonstrated that lines $\mathrm{Tg}(\mathrm{WT})$, $\operatorname{Tg}$ (mut1), and $\operatorname{Tg}$ (mut2) express human FBN1 mRNA in a quantity that is approximately equal to that expressed by WT human fibroblasts, while line $\operatorname{Tg}($ mut3) expresses approximately twice this amount (Figure 1B). This high-expressing line was used for further experimentation unless otherwise specified. Immunohistochemical analysis of cultured dermal fibroblasts using a mAb specific for human fibrillin-1 revealed the deposition of organized and abundant microfibrillar aggregates in all transgenic lines, including those harboring mutation C1663R (Figure 1C and data not shown). Cell lines derived from mice lacking the transgene did not show any signal, documenting the specificity of the Ab. These data provide the first evidence that cysteine-substituted forms of fibrillin- 1 are both bioavailable and competent to participate in microfibrillar assembly.

Transgenic overproduction of cysteine-substituted fibrillin-1 on a normal background has no phenotypic consequence. Mice harboring either the WT or mutant transgenes showed no phenotypic consequence for up to 2 years of age. Specifically, they were born at the expected Mendelian frequency, were fertile, and lived a normal life span as compared with WT littermates $(n>20$ for each genotype). Necropsy of mice that died naturally did not reveal any evidence of aortic dissection or internal hemorrhage. The aortic wall was examined at specific time points ( 6 months, 12 months, and 18 months) to assess for defined histologic end points that had been observed in previous mouse models of MFS, including elastic fiber fragmentation and calcification, accumulation of amorphous matrix elements, vascular smooth muscle cell (VSMC) proliferation, intimal hyperplasia, and inflammatory cell infiltration $(21,22,25)$. At least three mice for each strain and genotype were analyzed at each time point, including WT control mice derived from strain-specific littermates. The 12- month time point included at least five mice per genotype. No abnormalities in vessel wall architecture were observed (Figure $2 \mathrm{~A})$. Although the cardiovascular system was the major focus in this study, we noted the absence of any skeletal abnormalities, including the marked kyphosis and overgrowth of the ribs seen in previous mouse models of MFS (Figure 2B) (22).

$Y A C$-based transgenesis achieves normal regulation of fibrillin-1 expression in vivo. One possible explanation for the lack of apparent dominant-negative interference by the mutant human protein was that the transgene was not expressed in the appropriate tissues at critical developmental stages. To address this issue, an RT-PCR strategy was employed to both detect and quantify transgene-derived FBN1 message. Expression of the transgene in all strains was confirmed in late postnatal (embryonic day 17; ED 17) embryos. In addition, mRNA was isolated from hearts, lungs, aortae, kidneys, spleens, livers, tails, and brains from 2-month- and 6-month-old mice of each transgenic genotype with consistent results. Despite wide variation in the relative abundance of total fibrillin-1 message for each tissue and developmental stage, the estimated ratio of human-to-mouse mRNA was about 1:1 for the WT and mutant strains $\operatorname{Tg}($ mut 1$)$ and $\operatorname{Tg}($ mut 2$)$. Relative abundance of humanto-mouse mRNA in strain $\operatorname{Tg}$ (mut3) was about $2: 1$. Data from a representative organ (2-month aorta) is shown in Figure 3A.

Further evidence for proper regulation of transgene expression was developed through immunohistologic analysis of tissues derived from the $\mathrm{Tg}$ (mut3) line. For all tissues examined, the signal achieved with a $\mathrm{mAb}$ specific for human fibrillin-1 showed nearcomplete overlay with the signal using a polyclonal $\mathrm{Ab}$ that efficiently recognizes both mouse and human fibrillin-1 (Figure 3B and data not shown). Patchy excess of the mAb signal likely reflects differential affinities of the two Ab's and relative overexpression of the mutant human protein. This confirms transgene expression in tissues that physiologically express fibrillin-1. Taken together, these data suggest that use of YAC-based transgenesis has achieved sufficient temporal and spatial control of gene expression to assess the dominant-negative model of disease pathogenesis. 
A

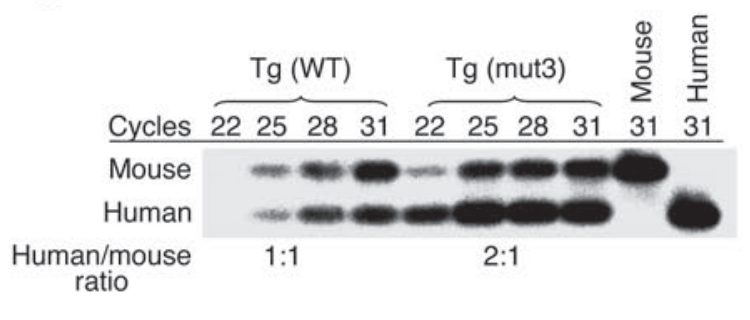

B

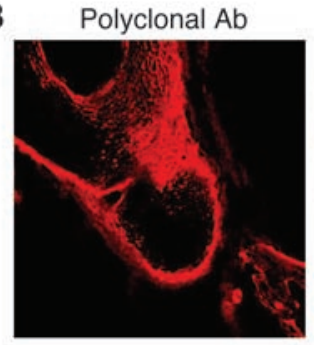

$\mathrm{mAb}$

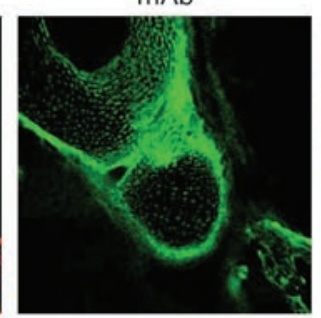

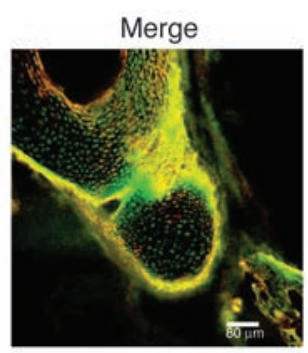

\section{Figure 3}

(A) Relative quantification of YAC transgene expression compared with endogenous Fbn1 expression. RT-PCR amplicons were generated using total RNA isolated from mouse aorta and oligonucleotides complementary to exons 10 and 11 that recognize identical sequence in mice and humans. The amplicon from FBN1 has a unique Taql digest site, resulting in a smaller band that binds to a probe generated from ${ }^{32} \mathrm{P}-\mathrm{labeled}$ antisense oligonucleotide in exon 11. Ratio of human to mouse mRNA in the exponential portion of amplification is indicated below. $\mathrm{Tg}$ (mut3) is the highest expressing strain, while two other strains were intermediate in their FBN1 expression. (B) Developing limb from Tg(mut3) mouse stained for fibrillin-1, demonstrating robust transgenic production of mutant human fibrillin-1 in tissues expressing endogenous mouse fibrillin-1. Left panel: polyclonal $\mathrm{Ab}$ that detects both mouse and human fibrillin-1; middle panel: $\mathrm{mAb}$ that is specific to human fibrillin-1; right panel: overlay.

Mouse and buman fibrillin-1 interact with high efficiency. The highsequence similarity between mouse and human fibrillin-1 (96\% amino acid identity) strongly supported the hypothesis that the proteins would interact and coassemble into microfibrils. Indeed, previous coculture experiments performed by Gayraud and colleagues provided significant evidence that human WISH cell-derived fibrillin-1 copolymerizes with a centrally duplicated form of fibrillin-1 expressed by mutant Tsk mice (26). Nevertheless, we felt compelled to demonstrate further evidence for interaction between mouse and human fibrillin-1 before interpreting the absence of an apparent dominant-negative effect. First, immunoelectron microscopy with a human-specific mAb was used to detect the diffuse and abundant incorporation of human fibrillin-1 in the microfibrillar matrices of developing skin and aorta of transgenic mice (Figure 4A and data not shown). As more direct proof, ${ }^{35} \mathrm{~S}$-labeled WT murine fibrillin-1 was added to the media of cultured human cells expressing fibril- lin-1. Immunoprecipitation of conditioned media with a $\mathrm{mAb}$ that specifically recognizes human fibrillin-1 coimmunoprecipitated ${ }^{35}$ S-labeled murine fibrillin-1 (Figure 4B). We concluded that overproduction of mutant fibrillin-1 that copolymerizes with mouse fibrillin- 1 in the critical tissues and developmental stages is insufficient to produce the clinical or aortic histologic features previously observed in fibrillin-1-deficient mice.

Heterozygous targeting of a missense mutation to an endogenous Fbn1 allele recapitulates manifestations of MFS. Next we sought to demonstrate that heterozygosity for a missense allele is sufficient to initiate phenotypic manifestations in mouse models. This was important since all previous models of MFS relied upon homozygosity for hypomorphic alleles $(21,22)$. Homologous recombination in murine ES cells was used to target mutation $\mathrm{C} 1039 \mathrm{G}$ into exon 25 of the mouse Fbn 1 gene that encodes a cbEGF-like domain (Figure 5A). This mutation was selected because it introduces a KpnI restriction site useful for

\section{Figure 4}

(A) Immunoelectron microscopy of mouse skin using an mAb that is specific for human fibrillin-1. Left panel, nontransgenic (NonTg) control, shows no staining, confirming previously described specificity of this Ab. Middle panel, $\operatorname{Tg}(\mathrm{WT})$, and right panel, $\mathrm{Tg}$ (mut3), both show Ab recognition of human fibrillin-1 in a pattern that suggests the presence of both murine and human proteins within the same microfibrillar bundle. Box in the lower-right corner highlights the pattern of epitope staining, seen in black. (B) Murine and human fibrillin-1 interact. Lanes 1 and $4:{ }^{35}$ S-labeled media from murine fibroblasts coincubated with unlabeled media from murine fibroblasts before and after immunoprecipitation (IP), respectively; lanes 2 and 5: identical treatment of ${ }^{35}$ S-labeled media from murine fibroblasts coincubated with unlabeled media from human fibroblasts before and after IP, respectively; lanes 3 and 6: identical treatment of ${ }^{35}$ S-labeled media from human fibroblasts coincubated with unlabeled media from human fibroblasts before and after IP, respectively. Results in lane 5 demonstrate interaction between ${ }^{35} \mathrm{~S}$-labeled murine fibrillin-1 and unlabeled human fibrillin-1, while results in lane 4 attest to the specificity of the mAb for human fibrillin-1. Lane 6 serves as a positive control for IP. Position of molecular weight standards is indicated on the left.
A

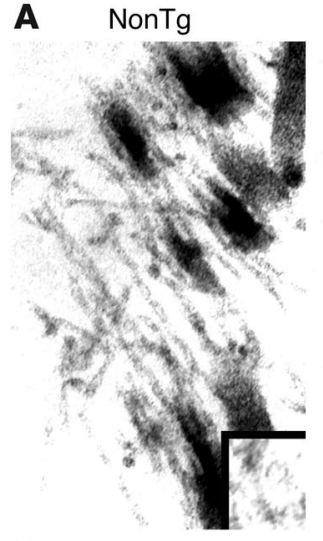

$\operatorname{Tg}(\mathrm{WT})$

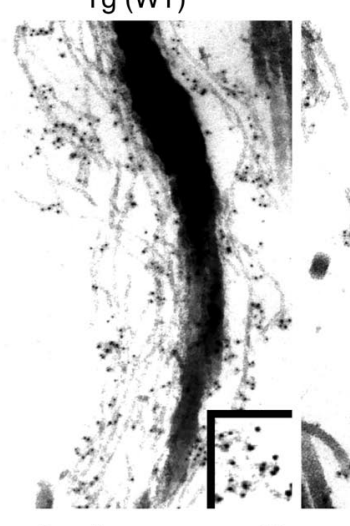

Tg (mut3)

B 
A C1039G:

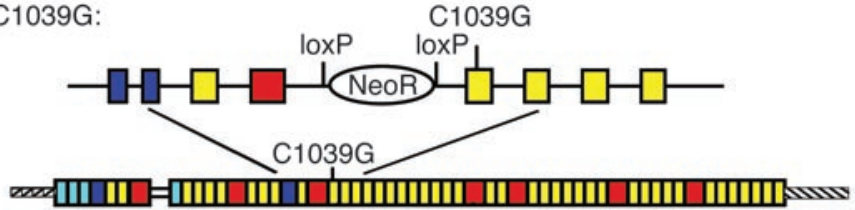

B

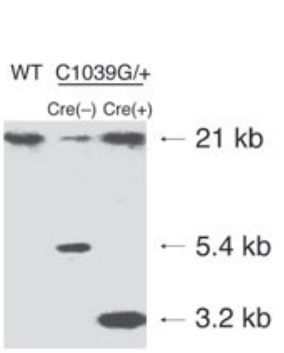

C

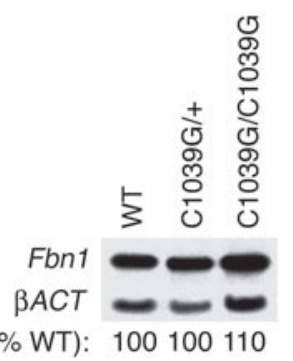

Figure 5

(A) Drawing depicting cDNA resulting from proper homologous recombination of the endogenous Fbn1 allele with the targeting vector for the C1039G mutation. Above is a representation of the genomic segment prior to Cre-mediated recombination to remove the NeoR flanked by loxP sequences. (B) Representative Southern blot demonstrating correct targeting before and after Cre-mediated loxP recombination. Left lane is from a WT, untargeted mouse, with an undigested 21-kb Kpnl fragment; middle lane is from a C1039G heterozygous mutant mouse without Cre-recombinase, resulting in an untargeted band (21 $\mathrm{kb})$ and a targeted band $(5.4 \mathrm{~kb})$; right lane is from a C1039G heterozygous mutant mouse after Cre-mediated recombination, resulting in an untargeted band $(21 \mathrm{~kb})$ and a targeted band $(3.2 \mathrm{~kb})$. (C) Northern blot demonstrating that the level of expression of $F b n 1$ in mice heterozygous or homozygous for the $\mathrm{C} 1039 \mathrm{G}$ allele is unchanged in comparison with untargeted (WT) mice. Expression is normalized to expression of the gene encoding $\beta$-actin (BACT). The level of Fbn1 expression in heterozygous and homozygous targeted mice, relative to that seen in WT, is indicated.

genotyping, and substitution of the corresponding cysteine residue in humans (C1039Y) has been associated with classic MFS (24). Furthermore, prior pulse-chase analysis of dermal fibroblasts from a patient harboring a substitution at this residue revealed impaired fibrillin-1 deposition despite normal synthesis (24). The targeting vector encoded loxP sites flanking the NeoR, allowing Cre-recombinase-mediated cassette deletion upon breeding of mice heterozygous for the C1039G mutation to mice transgenic for an allele that ubiquitously expresses Crerecombinase. This manipulation protects against transcriptional interference imposed by the NeoR as a confounding variable. Southern blot analysis demonstrated correct Fbn1 allele targeting and successful deletion of the selection cassette (Figure 5B). Northern blot analysis of cultured dermal fibroblasts demonstrated normal expression of $F b n 1$ mRNA in mice heterozygous or homozygous for the targeting event (Figure 5C).

Cultured dermal fibroblasts were analyzed for production of fibrillin-1 by immunohistochemistry using a polyclonal antifibrillin-1 Ab and established protocols. Cells heterozygous or homozygous for the C1039G Fbn1 mutation consistently demonstrated histologic evidence for reduced deposition of microfibrils (Figure 6A), as observed in heterozygous patient samples, but not cells derived from mice harboring a mutant $F B N 1$ transgene (Figure 1C). These data suggest that haploinsufficiency for WT fibrillin-1, rather than the production of mutant fibrillin-1, may be the critical determinant of impaired microfibrillar deposition both in cell culture and in vivo.

Subsequent analyses focused upon phenotypic characterization of the aortic wall in heterozygous $F b n 1^{\mathrm{C} 1039 \mathrm{G} /{ }^{+}}$mice. Heterozygotes and WT littermates were sacrificed at defined time points (birth, 3.5 days, 6.5 days, 3 months, 6 months, 9 months, and 1 year). The aortae from $\mathrm{Fbn}^{\mathrm{C1} 1039 \mathrm{G} / \mathrm{+}}$ mice were normal in appearance until about 2 months of age. Thereafter, there was progressive deterioration within the medial layer, including elastic fiber fragmentation and disarray of VSMCs (Figure 6B) in association with excess expression of MMP-2 and -9 (data not shown). There was also gradual thickening of the aortic wall due to the excessive deposition of amorphous matrix and increase in the number of resident VSMCs. Staining with trichrome and Safranin-O revealed excessive deposits of collagen and proteoglycans, respectively (Figure 6B and data not shown). The aortic wall showed a normal number of elastic lamellae at both early and late time points. While elastic fiber calcification was occasionally observed, other findings in previous recessive models of MFS, such as intimal hyperplasia and aortic wall inflammation, were not seen. As another distinction, $F b n 1^{\mathrm{C1} 1039 \mathrm{G} / \mathrm{+}}$ mice show a normal life span and do not die from aortic dissection. Thus, despite the absence of a clinical end point in this genetic background, $\mathrm{Fbn}^{\mathrm{C} 1039 \mathrm{G} / \mathrm{+}}$ mice recapitulate many of the histologic end points previously observed in more severe mouse models of fibrillin-1 deficiency. Furthermore, $\mathrm{Fbn1}^{\mathrm{C1039G/+}}$ mice show gradual postnatal development of typical skeletal changes, including kyphosis and overgrowth of the ribs, (Figure 2B) and show distal airspace widening in the absence of inflammation or tissue destruction (data not shown), as seen in hypomorphic mouse models of MFS $(22,27)$.

Introduction of a WT FBN1 transgene rescues aortic phenotype in Fbn $1^{\mathrm{C} 1039 \mathrm{G} /+}$ mice. Our current model posits that a half-normal dosage of WT fibrillin-1 precludes matrix use of protein derived from both the WT and mutant alleles. In the presence of a normal complement of WT protein, as in $\mathrm{Tg}$ (mut3) mice, microfibrillar assembly and deposition proceeds efficiently despite the production of mutant fibrillin-1 that is equal in amount to (or even in excess of) WT fibrillin-1. A prediction of this dosage-dependent deposition model is that the addition of the $\mathrm{Tg}(\mathrm{WT})$ allele to $\mathrm{Fbn} 1^{\mathrm{C} 1039 \mathrm{G} /+}$ mice should, at least to an extent, rescue phenotype. Histologic and precise morphometric analysis of aortic wall architecture and thickness again documented vascular pathology in $\mathrm{Fbn}^{\mathrm{C} 1039 \mathrm{G} / \mathrm{+}}$ mice that was not observed in transgenic animals harboring the $\mathrm{Tg}(\mathrm{mut} 3)$ allele (Figure 6, C and D). Mice compound for the $F b n 1^{\mathrm{C} 1039 \mathrm{G} /+}$ and $\mathrm{Tg}(\mathrm{WT})$ genotypes showed full normalization of this parameter. Intriguingly, even $F b n 1^{\mathrm{C} 1039 \mathrm{G} /+}$ mice harboring the $\mathrm{Tg}$ (mut3) allele showed relative improvement, suggesting that cysteine-substituted fibrillin-1 retains some residual function. While full details will be provided in a subsequent article, mice homozygous for the C1039G mutation uniformly die from vascular catastrophe in the perinatal period, a fate indistinguishable from that of mice homozygous for severe hypomorphic alleles. Furthermore, cells derived from these mice show a variable but significant impairment in microfibrillar deposition (Figure 6A). These data document a profound loss of function of fibrillin-1 harboring the C1039G mutation.

\section{Discussion}

Since the discovery that FBN1 mutations cause MFS, the pathogenesis of the disorder has been the subject of intense investigation and controversy (4). The Marfan phenotype is developmental- 
A
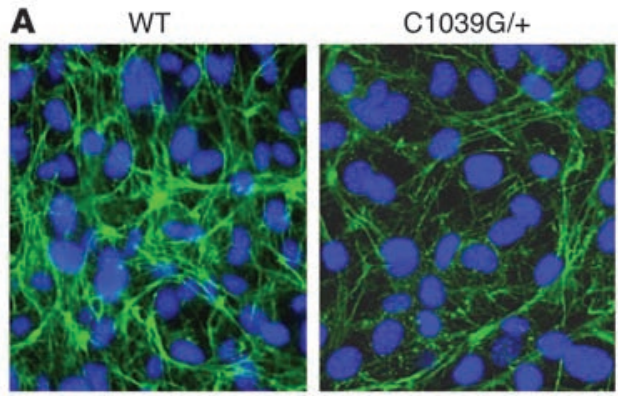

B
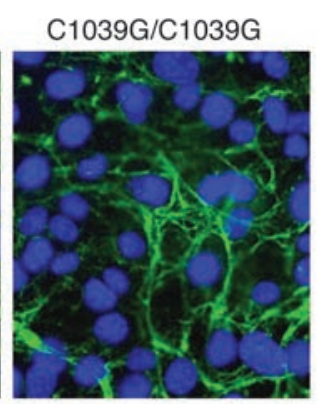

c

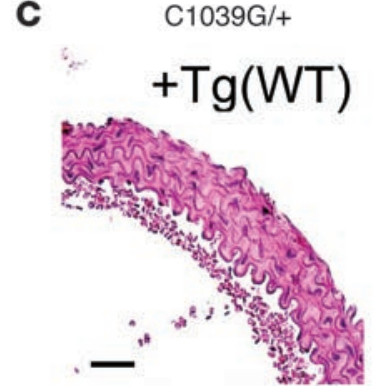

D

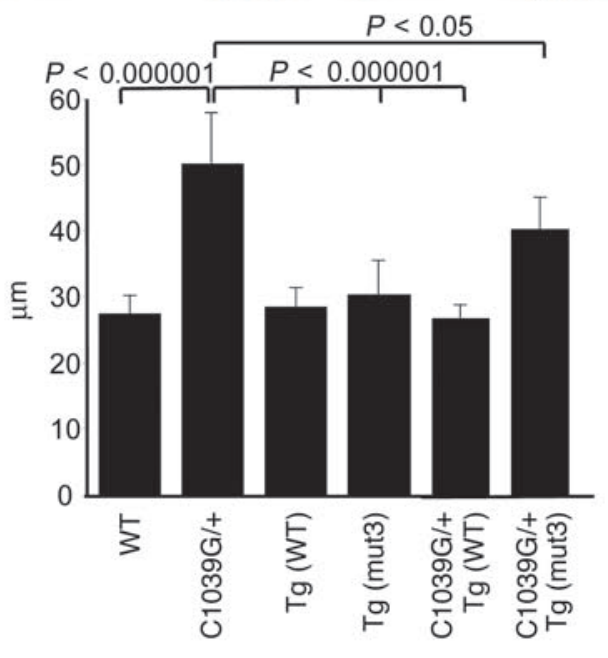

\section{。}
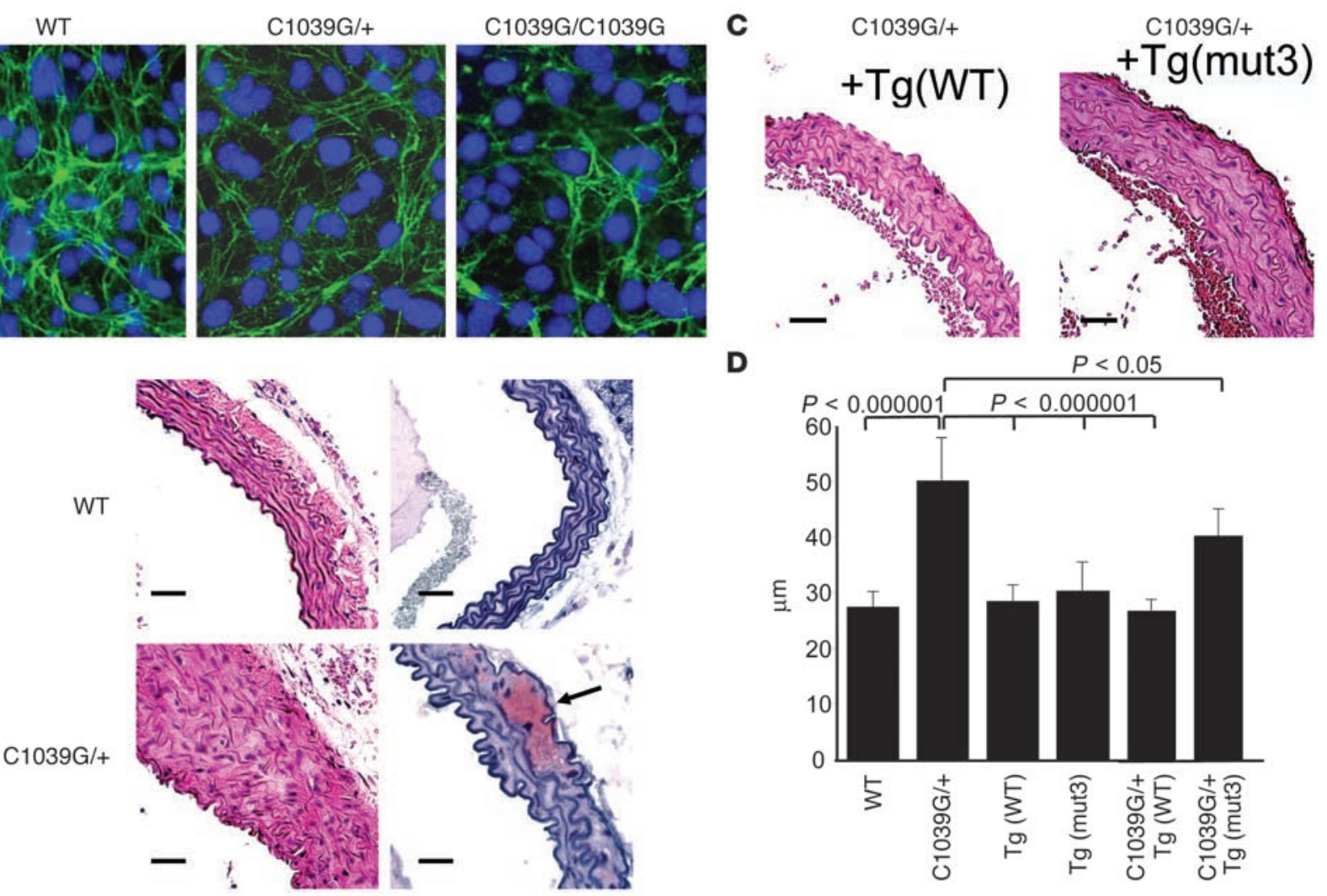

\section{Figure 6}

(A) Cysteine substitution in fibrillin-1 results in impaired microfibril formation. Cultured dermal fibroblasts from WT, heterozygous (C1039G/+), or homozygous (C1039G/C1039G) mice (for the Fbn1 mutation) were plated at $5 \times 10^{5} / \mathrm{ml}$ and immunostained for fibrillin-1 using polyclonal anti-fibrillin-1 Ab 9543 after 72 hours of confluence. The quantity and quality of the microfibrils containing the product of the mutant allele appears diminished. (B) Representative aortic wall sections from mice aged 6 months, stained with H\&E (left) and Safranin-O (right). Top panels are from a WT control, and bottom panels are from a mouse heterozygous for the C1039G mutation (C1039G/+). Mutant mice have focal areas of medial thickening with disorganization and disruption of elastic lamellae, as well as frequent areas of proteoglycan deposition (arrow). Magnification, $\times 40$ for all sections. Scale bar: $25 \mu \mathrm{m}$. (C) Representative aortic wall sections from mice heterozygous for the C1039G mutation and harboring the WT FBN1 transgene, $+\mathrm{Tg}(\mathrm{WT})$, or the C1663R-mutant FBN1 transgene, $+\mathrm{Tg}$ (mut3). Aortic wall organization is greatly improved by the WT transgene and partly improved by the mutant transgene. Magnification, $\times 40$ for all sections. Scale bar: $25 \mu \mathrm{m}$. (D) Average aortic wall thickness $(\mu \mathrm{m})$ from at least five mice of each genotype at age 6 months. For each mouse, at least six independent sections were examined, and for each section, four measures of the aortic wall thickness were averaged, including the thickest and thinnest, for each section.

ly acquired and manifests the dynamic interplay between primary tissue predisposition, physiologic stress, time, and both productive and deleterious compensatory events. This and previous studies attempt to mimic this complexity using multiple strains of mice that harbor targeted fibrillin-1 gene (Fbn1) alleles $(21,22,25)$. Taken together, they represent the full range of molecular mechanisms and clinical severity characteristic of MFS.

Early histopathologic studies in MFS demonstrated fragmentation of elastic fibers with a decrease in elastin content in both the skin and medial layer of the aorta (28). Ultrastructural, biochemical, and immunohistologic analyses of forming elastic fibers revealed that the amorphous component is surrounded by a lattice of microfibrils during embryonic development $(7,29,30)$. The tight temporal and spatial link between the formation of microfibrillar aggregates and the subsequent deposition of amorphous elastin fueled speculation that microfibrils are required for primary formation of elastic fibers during embryogenesis (31). Another belief was that fibrillin-1 is strictly a structural constituent of connective tissue. The implication was that individuals affected with MFS are born with an obligate predisposition for postnatal tissue failure due to failed elastogenesis and an inherent loss of biomechanical integrity. This boded poorly for the development of productive treatment strategies. Both beliefs have now been challenged through characterization of fibrillin-1deficient mice. First, mice homozygous for a hypomorphic allele that expresses low levels of a centrally deleted fibrillin-1 monomer show normal elastin content and the ability to deposit organized linear elastic fibers during embryonic growth (21). Fragmentation and disarray of elastic fibers is a focal and acquired event that occurs in association with abnormal production of matrix-degrading enzymes by resident VSMCs and ultimate vessel wall inflammation $(22,25)$. Second, it has been demonstrated that fibrillin-1 and microfibrils contribute to the regulated activation of members of the transforming growth factor- $\beta$ (TGF- $\beta$ ) superfamily of cytokines (27). Selected manifestations of MFS, including progressive pulmonary disease, manifest excessive cytokine activation and signaling, as evidenced by phenotypic rescue upon TGF- $\beta$ neutralization (27). Thus, there appear to be multiple therapeutic strategies aimed at blocking secondary events that could hold promise for the treatment of MFS. 
The most effective means for primary prevention of phenotypic manifestations of MFS would be to retain or restore microfibrillar abundance and function. Although in theory this might be achieved using pharmacologic or gene-transfer strategies, enthusiasm was low given the evidence for a traditional dominant-negative pathogenetic mechanism. Indeed, the apparent lesson from the study of patients harboring nonsense alleles suggested that mutant protein in extremely low abundance had potent dominantnegative potential that would neutralize even highly efficient strategies to boost the amount of WT fibrillin-1. The favored model of microfibrillar assembly suggests that fibrillin-1 monomers selfassemble into macroaggregates characterized by parallel arrays of linear extended structures with a head-to-tail orientation $(32,33)$. In this scenario, it is easy to imagine how the incorporation of relatively few mutant monomers would be sufficient to cause global perturbation of microfibrillar assembly and function.

Data presented here suggest that reduced dosage of normal fibrillin-1, rather than the production of mutant protein, is largely responsible for primary failure of matrix deposition of fibrillin-1 and productive microfibrillar assembly in mouse models. A threshold fibrillin-1 dosage dependence for microfibrillar assembly is supported by observations in the literature. First, immunohistochemical analysis of cultured dermal fibroblasts revealed that, despite ongoing transcription and fibrillin-1 translation in low-density cell cultures, microfibrillar deposition occurs as an apparent threshold event that correlates with fibrillin-1 concentration and/or cellular confluence (13). Second, pulse-chase analyses have demonstrated a consistent and significant time lag between fibrillin-1 synthesis and the onset of matrix deposition (34-36). The critical events that regulate deposition are poorly understood, but may include binding of fibrillin-1 monomers to heparin/heparan sulfate glycosaminoglycans at the cell surface (37). Competition with or direct inhibition of this interaction in cell culture systems resulted in a dramatic reduction of matrixassembled microfibrils despite normal protein synthesis and secretion, suggesting that saturation of heparan sulfate chain-binding may contribute to the early coordination of macroaggregate formation (37). The molecular basis for the apparent lack of ability of cysteine-substituted fibrillin-1 to efficiently cooperate with WT protein in early nucleation events remains unknown. In theory, this could relate to the delayed secretion of cysteine-substituted forms of fibrillin- 1 seen in pulse-chase analyses $(24,35)$ or to the anticipated consequence of such mutations on the local and longrange conformation of the protein (38).

The current data suggest that in the presence of a normal complement of WT protein, the production of cysteine-substituted fibrillin-1 becomes less relevant or even irrelevant to the pathogenesis of disease. It is important to avoid generalization of this observation to all mutant forms of fibrillin-1 in the absence of experimental validation. For example, multiple studies have demonstrated or implied that expression of isolated N-terminal peptides has the capacity to interfere with deposition of WT protein in cell culture systems $(11,39)$. Given our present data and the availability of improved experimental reagents, it seems advisable to revisit this issue using animal models.

If haploinsufficiency-imposed failure of microfibrillar assembly were the sole driving force in the pathogenesis of MFS, then it would be difficult to explain the wide range of clinical presentations seen in patients with MFS. The greatest variation is seen between families, including those harboring cbEGF-like domain cysteine substitutions. This observation is impossible to reconcile solely on the basis of genetic or environmental modifiers and strongly suggests the existence of additional genotype-modified events in the progression of disease. Susceptibility of established microfibrillar matrices to proteolytic degradation represents a strong candidate. For example, it has been shown that recombinant mutant fibrillin-1 peptides show enhanced proteolytic susceptibility when compared with their WT counterparts (40) and that the severity of this effect not only depends upon the type of mutation, but also its local context within the extended monomer (41). Calcium binding to EGF-like domains of fibrillin-1 protects the WT protein from the activity of proteases (42, 43). Mutations that either directly disrupt the calcium-binding consensus sequence in a given domain or that cause both local and distributed perturbation of protein conformation (e.g., cysteine substitutions) could culminate in significant (albeit variable and genotype-specified) secondary clearance. In this view, most or all patients heterozygous for missense or nonsense mutations in fibrillin-1 may show decreased protein deposition due to a dosage-dependent inhibition of assembly. Resulting clinical severity, however, titrates the inherent susceptibility of the residual microfibrils to subsequent proteolytic degradation. Indeed, the concept that clinical expression of MFS requires a threshold loss of microfibrillar function and correlates in severity with the extent of this effect is easily illustrated by previous experience with an allelic series of Fbn1-targeted animals $(21,22)$. A natural extension of this hypothesis would be that our line expressing the mutant transgene on a normal background has accelerated turnover of microfibrils. The caveat is that given robust microfibrillar assembly, as observed in this line, the net effect never reaches the threshold loss of function needed for clinical expression in the lifetime of the animal. A second prediction from our model is that patients and experimental animals harboring nonsense alleles or heterozygous entire gene deletions should, on average, show milder manifestations of MFS than carriers of heterozygous missense mutations, but that the resultant phenotype could be especially amenable to modification by alternative sources of variation. For example, it was recently shown that a patient missing one entire FBN1 allele due to a genomic deletion had only mild and nonspecific manifestations of MFS in association with nearnormal synthesis and deposition of fibrillin-1 (19). Here it was posited that polymorphic high-expressing WT FBN1 alleles may be protective in this setting. Indeed, analysis of a family segregating a nonsense $F B N 1$ allele showed that, among mutation carriers, only those harboring a high-expressing second allele were clinically protected. Absence of such a protective allele may explain a second patient that we have observed with an entire FBN1 allele deletion who showed striking features of MFS as an infant (H.C. Dietz, unpublished data). Informatively, preliminary analysis has revealed that mice heterozygous for a null $F b n 1$ allele are clinically and histologically indistinguishable from C1039G heterozygotes (Francesco Ramirez, unpublished data).

In summary, we have shown that the determinants of microfibrillar abundance and function in MFS are complex and perhaps variable in their relative significance between cases. Nevertheless, the novel insight that haploinsufficiency for WT protein can be a significant factor should prompt consideration of additional therapeutic strategies aimed at boosting protein expression. The success of our rescue experiment in which the addition of a normal allele rescued aortic phenotype in C1039G 
heterozygotes offers the potential that gene-transfer strategies would be productive in patients with MFS. The normal clinical and histologic presentation of mice overexpressing mutant fibrillin- 1 on a normal genetic background also suggests that a pharmacologic strategy that achieves nonspecific upregulation of both WT and mutant protein expression would provide a similar benefit. Our mouse models of MFS syndrome provide an ideal system to test these hypotheses.

\section{Methods}

YAC mutagenesis. A yeast strain containing YAC 757H8 was obtained from Research Genetics Inc. (Huntsville, Alabama, USA) and subjected to alu fragmentation by transformation with pBP81 (44). A 600-kb fragment containing the entire FBN1 gene and $150 \mathrm{~kb}$ of $5^{\prime}$ and $50 \mathrm{~kb}$ of $3^{\prime}$ flanking sequence was transferred into yeast strain YF1040 (MATa ura3-52 lys2-801 ade2-101 $\operatorname{trp} 1 \Delta 63$ bis $3 \Delta 200$ leu $\left.2 \Delta 1 c y b 2^{R} c a n^{R} k a r 1 \Delta 15\right)$ by kar 1 mating and was screened by pulsed-field gel electrophoresis (45). YAC-ductants were selected by uracil and tryptophan prototrophy as well as cycloheximide resistance. YAC DNA was compared to human genomic DNA by Southern blot analysis using four overlapping cDNA probes. This confirmed the absence of rearrangement or deletion of FBN1 within the fragmented YAC. Amplification of human genomic DNA from a patient with a C1663R mutation in FBN1 was performed using intronic primers flanking exon 40 (20). Sequence analysis was performed to confirm this mutation and the absence of any other mutations in the amplified portion of DNA. Two-step gene replacement was performed as previously described to introduce the mutation into the YAC (45). Sequence analysis of PCR amplicons derived from the resulting YAC revealed the presence of mutation C1663R.

YAC transgenesis. YAC DNA was transfected into murine ESJ1 cells (generously provided by J. Gearhart, Johns Hopkins University School of Medicine) using Lipofectamine (Invitrogen Corp., Carlsbad, California, USA), and stably transfected clones were selected for G418 resistance. ES clones were frozen prior to differentiation, and DNA was analyzed by PCR using primers specific to intronic sequence flanking exons 1,2 , and 65 . Clones that contained the FBN1 gene were thawed, and a portion was allowed to grow to confluence for Western blot analysis with a mAb specific for human fibrillin-1, as previously described (46), while the remainder was refrozen prior to differentiation. Positive clones were injected into C57B16/J blastocysts at ED 3.5 and transferred into pseudopregnant females. Chimeric offspring were mated to C57B16/J mice, and germline transmission was evident in three mutant FBN1 strains and one WT FBN1 strain. At least three successive backcrosses into C57B16/J mice were performed prior to all experiments, and control mice were littermates of the experimental mice. All animal experiments were approved by the Johns Hopkins Animal Care and Use Committee.

Genotyping of YAC transgenic mice. Presence of the human FBN1 transgene was assessed by PCR amplification using genomic DNA and primers fully complementary to both human FBN1 and murine Fbn1 (M10-S: ATTTCAACAAGCTGTGCTCTG; M11AS: ATTTTGACAGAGATAGCGGAC). Thirty cycles of PCR were performed $\left(94^{\circ} \mathrm{C}\right.$ for 30 seconds, $58^{\circ} \mathrm{C}$ for 30 seconds, $72^{\circ} \mathrm{C}$ for 60 seconds), and the amplicon was digested with restriction enzyme Asp718. Digestion products were separated on a $2 \%$ agarose gel. The murine amplicon results in both a 700-bp band and a 180bp band. The presence of the human YAC transgene was deter- mined on the basis of a lack of this restriction site in intron 10, with a single 880-bp band, in addition to the bands obtained from the endogenous murine Fbn1. The WT transgene was differentiated from the C1663R mutant transgene by PCR amplification of human exon 40 from the FBN1-containing YAC. PCR primers are based on the intronic sequence flanking exon 40 (40S: AAATGTGAAGTTTTCATATTCAC; 40AS: CATGCATTACTGAGAAAAGCT). Thirty cycles of PCR were performed $\left(94^{\circ} \mathrm{C}\right.$ for $30 \mathrm{sec}-$ onds, $58^{\circ} \mathrm{C}$ for 30 seconds, $72^{\circ} \mathrm{C}$ for 90 seconds), and the amplicon was digested with restriction enzyme Nla-III. Digestion products were separated on a $2 \%$ agarose gel. The $1663 \mathrm{R}$ mutation results in the loss of an Nla-III site. Repeat genotyping of transgenic strains was performed at regular intervals to confirm the presence and genotype of the YAC transgene.

Quantification of transgene $m R N A$ expression. Total RNA was extracted from mouse aorta, lung, heart, brain, liver, spleen, tail, and kidney using TRIzol (Invitrogen Corp.). Reverse transcription was performed using oligo-dT primers and the SuperScript FirstStrand Synthesis System (Invitrogen Corp.). PCR amplification was performed using primers M10S and M11AS, both of which are fully complementary to mouse and human cDNA. The human transcript was discerned from the murine transcript on the basis of a TaqI restriction site, which is not present in mouse-derived cDNA amplicons. Digestion products were separated on a $2 \%$ NuSeive 3:1 agarose gel (FMC Bioproducts, Rockland, Maine, USA). Varying cycle-number amplifications were performed for $15-35$ cycles to determine the region of exponential increase of each amplicon. After electrophoresis, the digestion products were Southern blottransferred to GeneScreen Plus nylon membranes (PerkinElmer Life and Analytical Sciences Inc., Boston, Massachusetts, USA) as previously described (47). Hybridization was performed using a ${ }^{32} \mathrm{P}-\boldsymbol{\gamma}$-dATP-labeled M11AS oligonucleotide. Relative quantification was determined using an Instant Imager (PerkinElmer Life and Analytical Sciences Inc.).

Northern blot analysis. Total RNA was extracted from either cultured dermal fibroblasts or whole mouse organs using TRIzol reagent (Invitrogen Corp.) and was electrophoresed on 1\% agarose, $15 \%$ formaldehyde gels using $1 \times$ Mops buffer. RNA was transferred to GeneScreen Plus nylon membranes (PerkinElmer Life and Analytical Sciences Inc.) as previously described (48). Probes unique to the genes encoding either murine or human fibrillin-1 were constructed using PCR products amplified from each respective $3^{\prime}$ UTR and labeled using ${ }^{32} \mathrm{P}-\alpha-\mathrm{dCTP}$ and the random-primed DNA labeling kit (Roche Applied Science, Indianapolis, Indiana, USA). Relative quantification was determined using an Instant Imager (PerkinElmer Life and Analytical Sciences Inc.).

Histology and immunohistochemistry. Cultured dermal fibroblasts at passages 3-5 obtained from neonatal mice were plated in six-well plates on glass coverslips (VWR International Inc., West Chester, Pennsylvania, USA) at a concentration of $5 \times 10^{5}$ cells $/ \mathrm{ml}$ and grown to confluence. Three days after confluence, cells were fixed for 10 minutes with $20^{\circ} \mathrm{C}$ acetone. After rehydration with PBS, the transgenic cells were incubated for 3 hours with mouse $\mathrm{mAb} 201$, and the nontransgenic C1039G cells were incubated for 3 hours with rabbit polyclonal $\mathrm{Ab}$ directed against fibrillin-1 (pAb 9543), as previously described $(46,49)$. Cells were washed with PBS and then incubated for 30 minutes with FITCconjugated goat anti-mouse or goat anti-rabbit IgG (Santa Cruz Biotechnology Inc., Santa Cruz, California, USA). Nuclei were stained with either DAPI or propidium iodide for 5 minutes, 
and inverted coverslips were mounted onto glass slides using Cytoseal (Vector Laboratories, Burlingame, California, USA). Negative results using secondary Ab's alone documented lack of cross-reactivity with mouse tissues.

Mice were killed with an inhalation overdose of halothane (Sigma-Aldrich, St. Louis, Missouri, USA). Mouse aortae were fixed in $10 \%$ buffered formalin overnight. Proximal aortic sections were dissected, and transverse sections were arranged in $1.5 \%$ agar prior to paraffin embedding, as previously described (50). Slides were prepared using $10-\mu \mathrm{M}$ sections and were stained with $H \& E$, alizarin red for calcium, Verhoeff-van Gieson for elastic tissue, Safranin-O for proteoglycans, and trichrome for collagen. Sections shown are representative of at least five mice of the identical genotype and age. WT mice used as controls were littermates of $\mathrm{C} 1039 \mathrm{G}$ heterozygotes, $\mathrm{Tg}(\mathrm{WT})$, or $\mathrm{Tg}($ mut3) mice. Histologic analyses included loss of elastin content, elastic fiber fragmentation, excessive deposition of amorphous matrix, elastic fiber calcification, and infiltration of inflammatory cells. For each age, strain, and genotype, at least five mice were included. Aortic wall thickness was determined by microscopy using an Eclipse E400 (Nikon Inc., Melville, New York, USA) microscope, using direct comparison to a $25-\mu \mathrm{m}$ calibration standard included with each image. For each genotype and age, at least ten independent aortic sections were measured at four distinct sites, including the maximal and minimal thickness per section. Averages were determined for each mouse. Averages per genotype were also determined, and comparisons across genotypes were made using the Student's $t$ test.

Generation of mice with Fbn1 mutation C1039G. A murine genomic fragment containing Fbn1 was obtained from Research Genetics Inc., and a 7.2-kb KpnI fragment containing exons 21-26 was subcloned into plasmid pSL301 (Invitrogen Corp.). Site-directed mutagenesis was performed using the QuikChange mutagenesis kit (Stratagene Inc., La Jolla, California, USA), substituting glycine for cysteine at codon 1039 (thymine $\rightarrow$ guanine at nucleotide 3115 ). Exons contained within the targeting vector were assessed by sequence analysis. The NeoR was amplified from pEGFP-C1 (Invitrogen Corp.) using PCR primers containing an EcoRI-BamH1-loxP linker (sense) and a loxP-EcoRI linker (antisense). The amplicon was subcloned into pCR2.1-TOPO (Invitrogen Corp.). An EcoRI fragment containing the BamH1 restriction site, loxP sequence, $\mathrm{NeoR}$, and flanking loxP sequence was subcloned into a unique EcoR1 site in Fbn1 intron 24, and sequence of the loxP sites was confirmed by direct sequencing. The vector was linearized by a unique (NotI) site and electroporated into R1 ES cells. Positive clones were identified by Southern blot analysis, recognizing the insertion of a novel KpnI site induced by the C1039G mutation. Probes consisting of genomic DNA $5^{\prime}$ and $3^{\prime}$ to the targeting vector, but within a BamH1 fragment, confirmed proper targeting. Positive clones were injected into C57Bl6/J blastocysts at ED 3.5 and transferred into pseudopregnant females. Chimeric offspring were mated to C57B16/J mice, and germline transmission was evident in three offspring. All exons within the targeting vector were subsequently analyzed by RT-PCR and direct sequencing, using total RNA derived from a C1039G homozygous mouse. Mice were subsequently genotyped by Southern blotting on the basis of the new KpnI site in the correctly targeted mice, as well as by PCR. Primers used for amplification were: intron 24S (TTGTCCATGTGCTTTAAGTAGC) and intron 25AS (ACAGAGGTCAGGAGATATGC). Cor- rectly targeted mice were identified on the basis of the KpnI site introduced by the $\mathrm{C} 1039 \mathrm{G}$ mutation in the amplicon. At least three successive backcrosses into C57B16/J mice were performed prior to all experiments, and control mice were littermates of the experimental mice. The loxP-flanked NeoR was removed by mating the $F b n 1^{\mathrm{C} 1039 \mathrm{G} /{ }^{+}}$mice with a transgenic mouse that ubiquitously expresses Cre-recombinase using a CMV promoter. (CMV-Cre mice generously provided by Roger Reeves, Johns Hopkins University.) Loss of loxP-flanked NeoR was assessed by Southern blot analysis.

Coimmunoprecipitation. Mouse and human dermal fibroblast cultures were plated at a concentration of $5 \times 10^{5}$ cells $/ \mathrm{ml}$ and grown to confluence in complete medium (MEM containing 16\% FBS, $100 \mathrm{U} / \mathrm{ml}$ penicillin G sodium, $100 \mu \mathrm{g} / \mathrm{ml}$ streptomycin sulfate, and $250 \mathrm{ng} / \mathrm{ml}$ amphotericin B) using T-75 flasks. After 3 days, cells were incubated for 30 minutes in $15 \mathrm{ml}$ DMEM lacking both cysteine and methionine (Invitrogen Corp.). Six flasks containing mouse fibroblasts and one containing human fibroblasts were pulse labeled with $5 \mu \mathrm{Ci}{ }^{35} \mathrm{~S}$-cysteine/methionine (Amersham Biosciences, Piscataway, New Jersey, USA) for 30 minutes. Labeling medium was then removed by aspiration, and cells were washed three times successively with $15 \mathrm{ml}$ of PBS and incubated with $2 \mathrm{ml}$ DMEM for 2 hours. Simultaneously, two flasks containing human fibroblasts and one containing mouse fibroblasts, which had been grown to confluence 3 days previously in complete medium, were washed three times with PBS and incubated for 2 hours in DMEM alone. Medium $(6 \mathrm{ml})$ from three flasks containing labeled mouse fibroblasts was removed and added to one flask containing human unlabeled fibroblasts. Medium $(6 \mathrm{ml})$ from the other three flasks containing labeled mouse fibroblasts was removed and added to a flask containing mouse unlabeled fibroblasts, and medium $(2 \mathrm{ml})$ from the flask containing labeled human fibroblasts was removed and added to the flask containing human unlabeled fibroblasts. Incubation for 60 minutes at $37^{\circ} \mathrm{C}$ was performed with $1 \times$ Complete Mini Protease Inhibitor Cocktail (Roche Applied Science). Medium was removed from cells and subsequently maintained at $4{ }^{\circ} \mathrm{C}$. Two microliters of mouse IgG (Dako Corp., Carpinteria, California, USA) were added to each sample, and the specimens were nutated end-over-end for 60 minutes at $4{ }^{\circ} \mathrm{C} ; 50 \mu \mathrm{l}$ of protein G-Sepharose beads (Amersham Biosciences) were then added and nutated at $4^{\circ} \mathrm{C}$ for 45 minutes. The beads were then pelleted at $5,000 \mathrm{~g}$ for 5 minutes at $4{ }^{\circ} \mathrm{C}$. The supernatants were transferred to new flasks, and $2 \mu \mathrm{l}$ of $\mathrm{mAb}$ to human fibrillin-1 (mAb 201) were added; the solutions were nutated for 120 minutes at $4^{\circ} \mathrm{C}$. Fifty microliters of protein G-Sepharose beads (Amersham Biosciences) were added and nutated at $4^{\circ} \mathrm{C}$ for 45 minutes. The beads were pelleted at $5,000 \mathrm{~g}$ for 5 minutes at $4^{\circ} \mathrm{C}$, and the supernatant was removed by aspiration. Beads were washed successively with buffers containing $50 \mathrm{mM}$ HEPES, $40 \mathrm{mM} \mathrm{NaCl}, 1 \%$ Triton X-100, followed by $50 \mathrm{mM}$ HEPES, $40 \mathrm{mM} \mathrm{NaCl}, 1 \%$ Triton X-100, and $500 \mathrm{mM}$ $\mathrm{LiCl}$. After washing, beads were pelleted at $5,000 \mathrm{~g}$ for 5 minutes at $4{ }^{\circ} \mathrm{C}$, and the supernatant was removed by aspiration. Beads were then incubated with $30 \mu \mathrm{l}$ SDS loading buffer containing -mercaptoethanol for 5 minutes at $95^{\circ} \mathrm{C}$, and samples were electrophoresed on a 3-8\% gradient Tris-acetate polyacrylamide gel (Novex; Invitrogen Corp). Gels were fixed (30\% methanol, 10\% acetic acid), dried, and exposed to film.

Radiography. Whole-body radiographs were taken using Kodak film (Eastman Kodak Co., Rochester, New York, USA) after eutha- 
nizing mice with $4 \%$ halothane by inhalation. Film was developed in a Kodak processor and scanned into Adobe Photoshop 6.0 (Adobe Systems Inc., San Jose, California, USA).

Light and EM. Light and EM of tissue samples were performed as previously described (51). Immunoelectron microscopy was performed using pAb 9543 and mAb 201 as previously described (49).

\section{Acknowledgments}

This study was supported by funding from the National Marfan Foundation; the William S. Smilow Center for Marfan Syndrome Research; the Dana and Albert "Cubby" Broccoli Center for Aortic
Diseases; NIH grants HL-067056 (D.P. Judge), AR-41135 and AR049698 (H.C. Dietz), and RR-00171 (D.L. Huso); the Howard Hughes Medical Institute; and the Shriners Hospitals for Children.

Received for publication November 25, 2003, and accepted in revised form May 4, 2004.

Address correspondence to: Harry C. Dietz, Johns Hopkins University School of Medicine, Ross Building, Room 858, 720 Rutland Avenue, Baltimore, Maryland 21205, USA. Phone: (410) 614-0701; Fax: (410) 614-2256; E-mail: hdietz@jhmi.edu.
1. Pyeritz, R.E. 1997. Marfan syndrome and other disorders of fibrillin. In Principles and practice of medical genetics. D.L. Rimoin, J.M. Connor, and R.E. Pyeritz, editors. Churchill Livingstone. New York, New York, USA

2. Gray, J.R., et al. 1994. Ascertainment and severity of Marfan syndrome in a Scottish population. J. Med. Genet. 31:51-54.

3. Silverman, D.I., et al. 1995. Life expectancy in the Marfan syndrome. Am. J. Cardiol. 75:157-160.

4. Dietz, H.C., et al. 1991. Marfan syndrome caused by a recurrent de novo missense mutation in the fibrillin gene. Nature. 352:337-339.

5. Dietz, H.C., et al. 1995. The question of heterogeneity in Marfan syndrome. Nat. Genet. 9:228-231.

6. Kainulainen, K., et al. 1991. Marfan syndrome: no evidence for heterogeneity in different populations, and more precise mapping of the gene. Am.J. Hum. Genet. 49:662-667.

7. Sakai, L.Y., Keene, D.R., and Engvall, E. 1986. Fibrillin, a new $350-\mathrm{kD}$ glycoprotein, is a component of extracellular microfibrils. J. Cell Biol. 103:2499-2509.

8. Godfrey, M., et al. 1990. Cosegregation of elastinassociated microfibrillar abnormalities with the Marfan phenotype in families. Am. J. Hum. Genet. 46:652-660.

9. Hollister, D.W., Godfrey, M., Sakai, L.Y., and Pyeritz, R.E. 1990. Immunohistologic abnormalities of the microfibrillar-fiber system in the Marfan syndrome. N. Engl. J. Med. 323:152-159.

10. Godfrey, M., et al. 1990. Unilateral microfibrillar abnormalities in a case of asymmetric Marfan syndrome. Am. J. Hum. Genet. 46:661-671.

11. Eldadah, Z.A., Brenn, T., Furthmayr, H., and Dietz, H.C. 1995. Expression of a mutant human fibrillin allele upon a normal human or murine genetic background recapitulates a Marfan cellular phenotype. J. Clin. Invest. 95:874-880.

12. Kielty, C.M., Phillips, J.E., Child, A.H., Pope, F.M., and Shuttleworth, C.A. 1994. Fibrillin secretion and microfibril assembly by Marfan dermal fibroblasts. Matrix Biol. 14:191-199.

13. Brenn, T., Aoyama, T., Francke, U., and Furthmayr, H. 1996. Dermal fibroblast culture as a model system for studies of fibrillin assembly and pathogenetic mechanisms: defects in distinct groups of individuals with Marfan's syndrome. Lab. Invest. 75:389-402.

14. Collod-Beroud, G., et al. 1998. Marfan database (third edition): new mutations and new routines for the software. Nucleic Acids Res. 26:229-233.

15. Frischmeyer, P.A., and Dietz, H.C. 1999. Nonsensemediated mRNA decay in health and disease. Hum. Mol. Genet. 8:1893-1900.

16. Hewett, D., Lynch, J., Child, A., Firth, H., and Sykes, B. 1994. Differential allelic expression of a fibrillin gene (FBN1) in patients with Marfan syndrome. Am. J. Hum. Genet. 55:447-452.

17. Dietz, H.C., et al. 1993. Four novel FBN1 mutations: significance for mutant transcript level and EGF-like domain calcium binding in the pathogenesis of Marfan syndrome. Genomics. 17:468-475.

18. Schrijver, I., et al. 2002. Premature termination muta- tions in FBN1: distinct effects on differential allelic expression and on protein and clinical phenotypes. Am.J.Hum. Genet. 71:223-237.

19. Hutchinson, S., et al. 2003. Allelic variation in normal human FBN1 expression in a family with Marfan syndrome: a potential modifier of phenotype? Hum. Mol. Genet. 12:2269-2276.

20. Nijbroek, G., et al. 1995. Fifteen novel FBN1 mutations causing Marfan syndrome detected by heteroduplex analysis of genomic amplicons. Am. J. Hum. Genet. 57:8-21.

21. Pereira, L., et al. 1997. Targeting of the gene encoding fibrillin-1 recapitulates the vascular aspect of Marfan syndrome. Nat. Genet. 17:218-222.

22. Pereira, L., et al. 1999. Pathogenetic sequence for aneurysm revealed in mice underexpressing fibrillin-1. Proc. Natl. Acad. Sci. U. S. A. 96:3819-3823.

23. Liu, W.O., Oefner, P.J., Qian, C., Odom, R.S., and Francke, U. 1997. Denaturing HPLC-identified novel FBN1 mutations, polymorphisms, and sequence variants in Marfan syndrome and related connective tissue disorders. Genet. Test. 1:237-242.

24. Schrijver, I., Liu, W., Brenn, T., Furthmayr, H., and Francke, U. 1999. Cysteine substitutions in epidermal growth factor-like domains of fibrillin-1: distinct effects on biochemical and clinical phenotypes. Am. J. Hum. Genet. 65:1007-1020.

25. Bunton, T.E., et al. 2001. Phenotypic alteration of vascular smooth muscle cells precedes elastolysis in a mouse model of Marfan syndrome. Circ. Res. 88:37-43.

26. Gayraud, B., Keene, D.R., Sakai, L.Y., and Ramirez, F. 2000. New insights into the assembly of extracellular microfibrils from the analysis of the fibrillin 1 mutation in the tight skin mouse. J. Cell Biol. 150:667-680.

27. Neptune, E.R., et al. 2003. Dysregulation of TGFbeta activation contributes to pathogenesis in Marfan syndrome. Nat. Genet. 33:407-411.

28. Abraham, P.A., Perejda, A.J., Carnes, W.H., and Uitto, J. 1982. Marfan syndrome. Demonstration of abnormal elastin in aorta. J. Clin. Invest. 70:1245-1252.

29. Greenlee, T.K.J., Ross, R., and Hartman, J.L. 1966. The fine structure of elastic fibers. J. Cell. Biol. 30:59-71.

30. Fahrenbach, W.H., Sandberg, L.B., and Cleary, E.G. 1966. Ultrastructural studies on early elastogenesis. Anat. Rec. 155:563-576.

31. Trask, T.M., et al. 2000. Interaction of tropoelastin with the amino-terminal domains of fibrillin- 1 and fibrillin-2 suggests a role for the fibrillins in elastic fiber assembly. J. Biol. Chem. 275:24400-24406.

32. Baldock, C., et al. 2001. The supramolecular organization of fibrillin-rich microfibrils. J. Cell Biol. 152:1045-1056.

33. Sakai, L.Y., Keene, D.R., Glanville, R.W., and Bachinger, H.P. 1991. Purification and partial characterization of fibrillin, a cysteine-rich structural component of connective tissue microfibrils. J. Biol. Chem. 266:14763-14770.

34. Trask, T.M., Ritty, T.M., Broekelmann, T., Tisdale, C., and Mecham, R.P. 1999. N-terminal domains of fibrillin 1 and fibrillin 2 direct the formation of homodimers: a possible first step in microfibril assembly. Biochem. J. 340:693-701.

35. Halliday, D., et al. 1999. Molecular analysis of eight mutations in FBN1. Hum. Genet. 105:587-597.

36. Raghunath, M., Superti-Furga, A., Godfrey, M., and Steinmann, B. 1993. Decreased extracellular deposition of fibrillin and decorin in neonatal Marfan syndrome fibroblasts. Hum. Genet. 90:511-515.

37. Tiedemann, K., Batge, B., Muller, P.K., and Reinhardt, D.P. 2001. Interactions of fibrillin-1 with heparin/heparan sulfate, implications for microfibrillar assembly. J. Biol. Chem. 276:36035-36042.

38. Handford, P., et al. 1995. The calcium binding properties and molecular organization of epidermal growth factor-like domains in human fibrillin-1. J. Biol. Chem. 270:6751-6756.

39. Guo, D., Tan, F.K., Cantu, A., Plon, S.E., and Milewicz, D.M. 2001. FBN1 exon 2 splicing error in a patient with Marfan syndrome. Am. J. Med. Genet. 101:130-134.

40. Hindson, V.J., et al. 1999. Fibrillin degradation by matrix metalloproteinases: identification of aminoand carboxy-terminal cleavage sites. FEBS Lett. 452:195-198.

41. Booms, P., Tiecke, F., Rosenberg, T., Hagemeier, C., and Robinson, P.N. 2000. Differential effect of FBN1 mutations on in vitro proteolysis of recombinant fibrillin-1 fragments. Hum. Genet. 107:216-224.

42. Reinhardt, D.P., Ono, R.N., and Sakai, L.Y. 1997. Calcium stabilizes fibrillin-1 against proteolytic degradation. J. Biol. Chem. 272:1231-1236.

43. Reinhardt, D.P., et al. 2000. Mutations in calciumbinding epidermal growth factor modules render fibrillin-1 susceptible to proteolysis. A potential disease-causing mechanism in Marfan syndrome. J. Biol. Chem. 275:12339-12345.

44. Pavan, W.J., Hieter, P., Sears, D., Burkhoff, A., and Reeves, R.H. 1991. High-efficiency yeast artificial chromosome fragmentation vectors. Gene. 106:125-127.

45. Spencer, F., et al. 1994. Yeast kar1 mutants provide an effective method for YAC transfer to new hosts. Genomics. 22:118-126.

46. Reinhardt, D.P., et al. 1996. Fibrillin-1: organization in microfibrils and structural properties. J. Mol. Biol. 258:104-116.

47. Southern, E.M. 1975. Detection of specific sequences among DNA fragments separated by gel electrophoresis. J. Mol. Biol. 98:503-517.

48. Montgomery, R.A., and Dietz, H.C. 1997. Inhibition of fibrillin 1 expression using U1 snRNA as a vehicle for the presentation of antisense targeting sequence. Hum. Mol. Genet. 6:519-525.

49. Charbonneau, N.L., et al. 2003. Fibrillins can coassemble in fibrils, but fibrillin fibril composition displays cell-specific differences. J. Biol. Chem. 278:2740-2749.

50. LoRusso, F.J., and Font, R.L. 1999. Use of agar in ophthalmic pathology: a technique to improve the handling and diagnosis of temporal artery biopsies, subfoveal membranes, lens capsules, and other ocular tissues. Ophthalmology. 106:2106-2108.

51. Sakai, L.Y., and Keene, D.R. 1994. Fibrillin: monomers and microfibrils. Methods Enzymol. 245:29-52. 